\title{
Properties of a vacuum ultraviolet laser created plasma sheet for a microwave reflector
}

\author{
W. Shen, J. E. Scharer, ${ }^{a)}$ N. T. Lam, B. G. Porter, and K. L. Kelly \\ Department of Electrical and Computer Engineering, University of Wisconsin-Madison, \\ Madison, Wisconsin 53706
}

(Received 16 March 1995; accepted for publication 5 September 1995)

\begin{abstract}
A $193 \mathrm{~nm}$ excimer laser and a custom fabricated cylindrical lens system is used to produce a plasma sheet of $8 \mathrm{~cm} \times 30 \mathrm{~cm} \times 0.4 \mathrm{~cm}$ in tetrakis(dimethylamino)ethylene (TMAE), a low ionization energy organic gas. Plasma density variation due to photon absorption is studied by scanning the filling pressure of TMAE between 12 and 150 mTorr. A high density $\left(n \geqslant 2.0 \times 10^{13} \mathrm{~cm}^{-3}\right)$, low temperature $\left(T_{e} \approx 0.8 \mathrm{eV}\right)$ plasma sheet of $4 \mathrm{~mm}$ thickness is obtained with less than $50 \%$ spatial density variation over the $30 \mathrm{~cm}$ axial length. Charge recombination is found to be the dominant process for $t \leqslant 1.2 \mu$ s with the plasma diffusion playing a perturbational role. A one-dimensional plasma model is utilized to model the experimental plasma data by treating the diffusion as a perturbation. This study shows that the recombination coefficient is $1.8 \pm 0.1 \times 10^{-7} \mathrm{~cm}^{3} \mathrm{~s}^{-1}$ and the diffusion coefficient is $2.8 \pm 0.4 \times 10^{4} \mathrm{~cm}^{2} \mathrm{~s}^{-1}$. The plasma sheet has attractive properties for a microwave agile mirror. (C) 1995 American Institute of Physics.
\end{abstract}

\section{INTRODUCTION}

There have been several studies ${ }^{1-3}$ of a plasma as a microwave reflector or absorber based on its application in the field of radar communications. The primary interest is to use a plasma as an agile reflector to reflect or cut-off radar pulses, to replace a complex phased-array radar system to steer a radar beam. The chief advantages of using a laser formed plasma sheet are its short turn on/off time, high reflection coefficient, and possible simplicity compared to the microwave hardware for high power phased-array radar systems. Recent experimental studies at the Naval Research Laboratory ${ }^{4}$ illustrate that a plasma sheet generated using a linear hollow cathode immersed in a magnetic field yields a reflected far-field microwave antenna pattern very close to that from a metal plate.

It has been observed from previous experiments ${ }^{5}$ in our laboratory that a plasma with a density above the critical density $\left(n \geqslant 1.2 \times 10^{12} \mathrm{~cm}^{-3}\right)$ for reflection of X-band microwaves can be produced by means of a photoionization mechanism in a low ionization energy organic gas. Compared with the experiment in Ref. 4 , the optically generated plasma can have a much higher plasma density $\left(n \approx 2.5 \times 10^{13}\right.$ $\mathrm{cm}^{-3}$ ), thus it could be used as an agile mirror working in even higher frequency regimes. In addition, the laser produced plasma sheet has a much faster turn-on and turn-off time $\left(\tau_{\mathrm{on}} \approx 10 \mathrm{~ns}, \tau_{\text {off }} \approx 1 \mu \mathrm{s}\right)$ compared with a hollow cathode system. The fast turn-on time also provides an attractive application as a radar beam shutter. Other advantages include the relative ease of adjustment of the sheet dimensions and the absence of a magnetic field requirement. Previous experimontal studies ${ }^{6}$ in our laboratory have made detailed measurements of plasma absorption, transmission, and backscattering in the $2-4 \mathrm{GHz}$ microwave frequency range.

In this article we report on an experiment which produces a plasma sheet with a vacuum ultraviolet (VUV) laser

${ }^{a)}$ Electronic mail: scharer@tesla.ece.wisc.edu in an organic gas using a cylindrical lens system for beam shaping. We also report studies on the spatial extent, diffusion, and recombination of the plasma sheet. Measurements of the plasma density and temperature, and their temporal and spatial variations are presented. Meanwhile, we have modeled plasma dynamics by comparing plasma quantities which are derived from measurement, such as the recombination and plasma diffusion coefficients, with predictions from a nonlinear diffusion equation.

The remainder of the article is organized as follows. In Sec. II we describe the experimental apparatus and diagnostics used. In Sec. III we present a detailed measurement of the laser produced plasma in an organic gas with changing experimental conditions. A plasma diffusion/recombination model is presented and derivation of plasma parameters based on this model is described in Sec. IV. Finally, conclusions from the studies are made in Sec. V.

\section{EXPERIMENTAL APPARATUS}

Figure 1 illustrates the basic experimental arrangement used to generate the plasma sheet. An excimer laser (Lambda Physik EMG-50E) source is used to produce a $193 \mathrm{~nm}$ VUV beam. The peak laser energy as measured by a calorimeter is $20 \mathrm{~mJ}$ for a $17 \mathrm{~ns}$ pulse. The laser output cross section is a $2.2 \mathrm{~cm} \times 0.7 \mathrm{~cm}$ rectangle. A two-to-four-element custom designed VUV coated cylindrical lens system with high transparency ( $98 \%$ per lens) at $193 \mathrm{~nm}$ is used to transform the laser beam cross section to a "sheet beam" of $1.5-4 \mathrm{~mm} \times 8$ $\mathrm{cm}$ rectangular cross section.

The plasma chamber is made of a 6-in.-diam Corning glass Tee with three openings. The cotal length of the chamber is $50 \mathrm{~cm}$ but the plasma sheet extent is typically about 30 $\mathrm{cm}$ long due to substantial photon absorption at moderate filling pressures. for high density $\left(n \approx 2.5 \times 10^{13} \mathrm{~cm}^{-3}\right)$ operation. A 6 in. supersil window that is coated for a $98 \%$ VUV transparency is mounted at the laser entrance end of the plasma vacuum chamber. Diagnostic probes, as well as a 


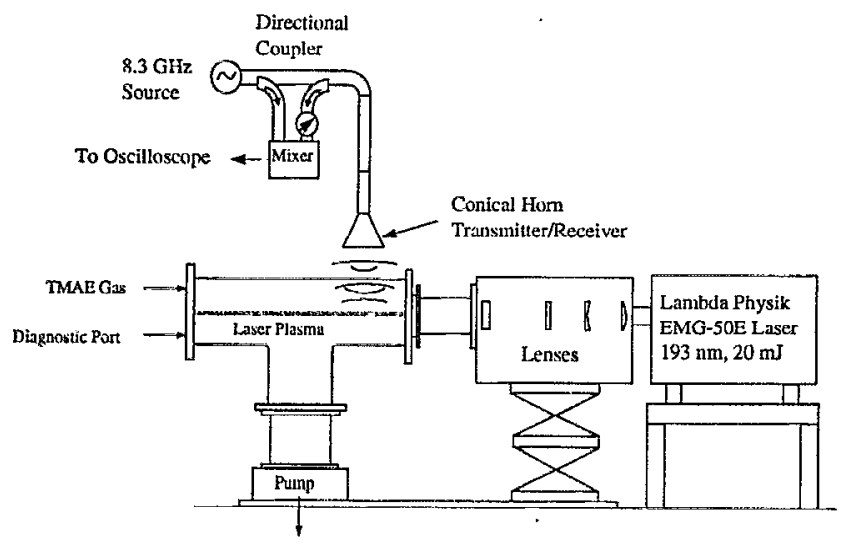

FIG. 1. The setup of the laser produced plasma experiment.

tetrakis(dimethylamino)ethylene (TMAE) gas flow feed, are inserted into the chamber from the opposite side. A needle valve controls the partial pressure of TMAE in the chamber for $P \leqslant P_{\text {vapor }}$ where $P_{\text {vapor }}$ is the vapor pressure of TMAE at room temperature. The third opening is connected to a vacuum pump system which consists of a mechanical rotary pump and a high vacuum diffusion pump. The plasma chamber is evacuated to $8 \times 10^{-6}$ Torr before filling with the working gas. The pre-evacuation is carried out to produce a high purity TMAE gas medium at pressures of $50-200 \mathrm{mTorr}$. We calibrated the cold cathode vacuum gauge against a McLeod gauge with the TMAE gas.

Langmuir probes are the main plasma diagnostic from which the results are obtained. The ion saturation current collected by a single-tip probe is related to the plasma density from probe theory for plasmas. The tantalum probe tip ( $2.4 \mathrm{~mm}$ in diameter) is biased at $-20 \mathrm{~V}$ during the measurement. The threshold for the ablation of tantalum by VUV radiation is on the order of $10^{8} \mathrm{~W} / \mathrm{cm}^{2}$. Our peak laser beam power density is measured to be less than $10^{6} \mathrm{~W} / \mathrm{cm}^{2}$ by means of an optical calorimeter. The photoelectron effect is, therefore, negligible in interpreting the probe signals. This was further verified by the experimental measurement. At $P=10^{-6}$ Torr, the detected Langmuir probe signals when the laser is fired are three orders of magnitude smaller when TMAE gas is absent. The plasma temperature is measured in a single shot using a triple-tip probe technique. ${ }^{7}$ The plasma density and temperature profiles are measured by moving the probes in two-dimensional space inside the chamber.

\section{SHEET PLASMA FORMATION BY THE PHOTON-IONIZATION PROCESS}

At room temperature $\left(20^{\circ} \mathrm{C}\right)$, the vapor pressure of TMAE is $350 \mathrm{mTorr}$ and its ionization energy is $5.36 \mathrm{eV}^{8}$ The photon energy of the $193 \mathrm{~nm}$ laser beam is $6.4 \mathrm{eV}$ so a photoionized plasma can be generated via a single photon absorption process. The laser pulse duration is $17 \mathrm{~ns}$. Use of a Tektronix TDS350 scope (1 GHz sample rate) shows (see Fig. 2) that the Langmuir probe measurement of plasma den-

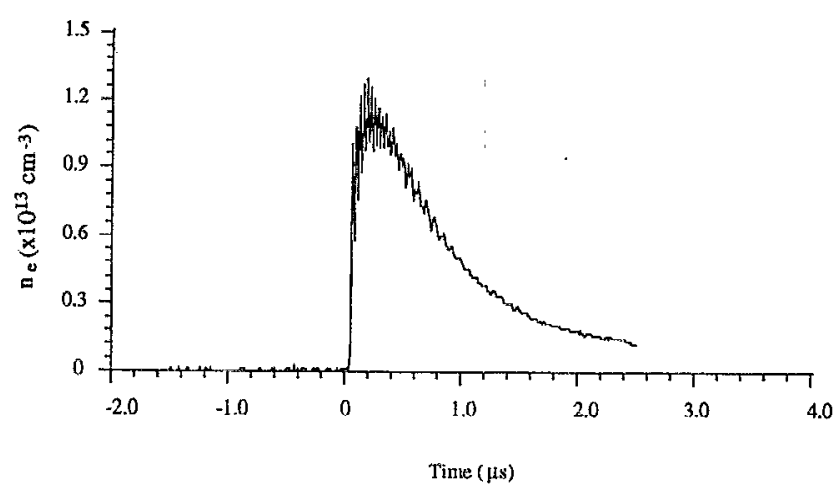

FIG. 2. Time evolution of the Langmuir probe signals (ion saturation current).

sity at $P=150$ mTorr reaches its peak $\left(n \approx 2.5 \times 10^{13} \mathrm{~cm}^{-3}\right)$ $50-100 \mathrm{~ns}$ after the laser pulse is off, followed by a slow decay mode for several $\mu \mathrm{s}$. Typically the plasma density remains above $10^{12} \mathrm{~cm}^{-3}$ for over $2 \mu \mathrm{s}$.

It is found that the laser pulse power can have fluctuations of $50 \%$ from one shot to another. To minimize the experimental uncertainty caused by this shot to shot laser power fluctuation on the plasma density profile measurements, a reference probe at a fixed position is installed. For profile measurements the ion saturation current data are first screened so that only the data within $25 \%$ of the peak plasma density shots are selected. The signals are then normalized to the values from the reference probe. The normalization procedure greatly reduces the systematic statistical deviation and provides better plasma profile information even though the laser power varies up to $50 \%$ from shot to shot.

\section{A. A window (10-150 mTorr) of TMAE filling pressure for sheet plasma formation}

To obtain a maximum plasma density at the laser beam entrance it is desirable to run the experiment at the room temperature vapor pressure (350 mTorr) for TMAE. However, we have observed that total absorption of the laser beam photon energy occurs within a length of $2 \mathrm{~cm}$ under these conditions. This is comparable with the result of a study by Anderson ${ }^{8}$ which states that the TMAE absorption length for broadband VUV radiation at room temperature vapor pressure is $1.2 \mathrm{~cm}$. To generate a plasma sheet with a substantial extension along the laser beam, we examined the dependence of the photon absorption length as a function of TMAE base pressure.

Figures 3(a) and 3(b) illustrate the plasma density profile along the laser beam at different TMAE filling pressures. The data are averaged over the first 100 ns after the density peak. Figure 3(a) shows that the higher the base pressure, the faster the plasma density attenuates, thus the shorter the laser absorption length is. The plasma density variation over a 30 $\mathrm{cm}$ distance is $\sim 80 \%$ for a $140 \mathrm{mTorr}$ pressure, and is $\sim 35 \%$ for $60 \mathrm{~m}$ Torr. Figure 3(a) also shows that the plasma density very near the entrance window is about the same for pressures of 150 and $60 \mathrm{mTorr}$, indicating a high photonionization coefficient. A lower base pressure thus is preferred 

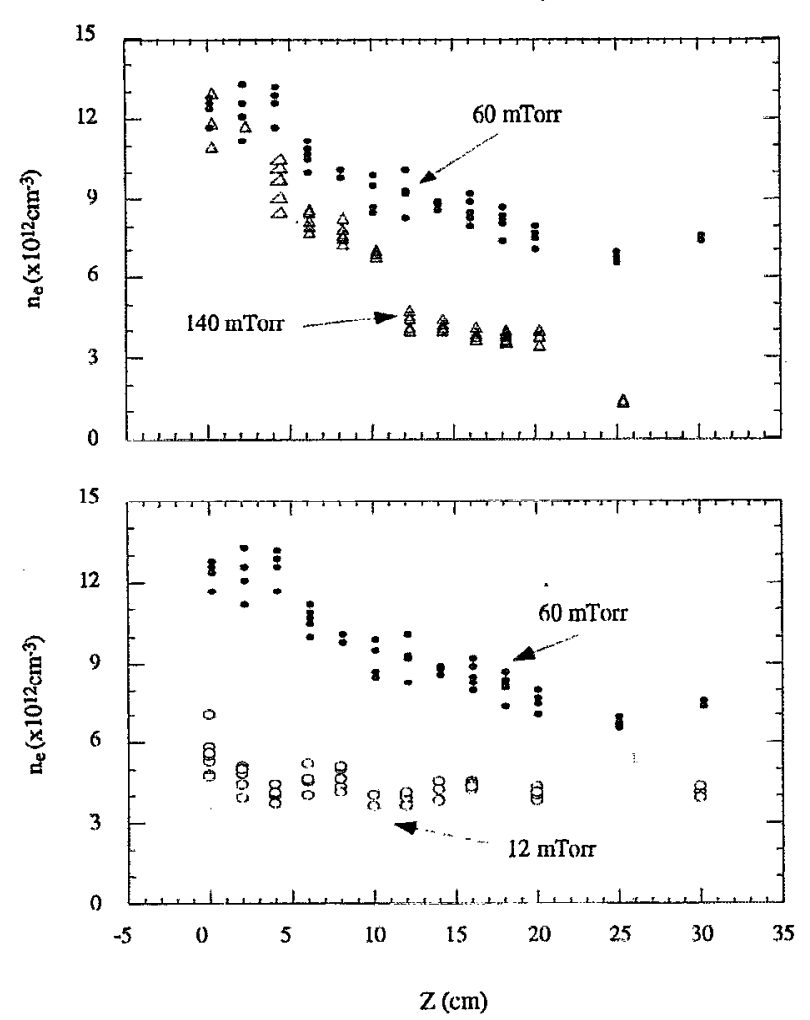

FIG. 3. Plasma density decay along the beam at different TMAE pressures.

for obtaining a more axially uniform plasma sheet provided that the overall plasma density is above the X-band critical density of $1.2 \times 10^{12} \mathrm{~cm}^{-3}$.

If the ionization process follows a single photon absorption process, the absorption length can be calculated simply from

$$
\frac{1}{\lambda}=-\frac{1}{x} \ln \left(\frac{I}{I_{0}}\right)=-\frac{1}{x} \ln \left(\frac{n}{n_{0}}\right),
$$

where $\lambda$ is the laser absorption length, $I_{0}$ and $n_{0}$ are the laser intensity and the plasma density at the entrance window $(x=0)$, and $I$ and $n$ are the laser intensity and the plasma density at a distance $x$ from the window.

From Eq. (1) we determine that $\lambda$ is $15 \mathrm{~cm}$ for $P=140$ m'Torr and $35 \mathrm{~cm}$ for $P=60 \mathrm{~m}$ Torr, respectively. Substituting $P$ into the simple gas model we obtain the "effective" photon absorption cross section of $\sigma=1.2 \times 10^{-17} \mathrm{~cm}^{2}$. This result is in good agreement with our previous measurements 5 using the output beam from the excimer laser source at a 200 mTorr filling pressure. To get the "true" photon absorption cross section one must include the quantum efficiency of ionization. ${ }^{9}$ Using the measured absorption length we calculated the extinction coefficient to be $7.7 \times 10^{3} \mathrm{~L} / \mathrm{mol} \mathrm{cm}$. This result is in good agreement with the results previously obtained for TMAE by Holroyd et al. ${ }^{9}$

When the base pressure decreases to $P=12 \mathrm{mTorr}$ [Fig. 3(b)], we find there is a strong reduction in peak plasma density, along with a very long absorption length. Over 30 $\mathrm{cm}$, the plasma density is constant within the measurement

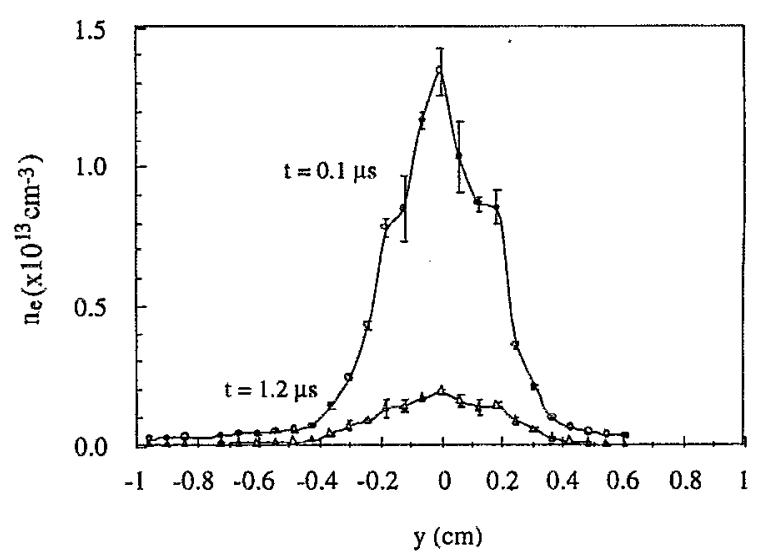

FIG. 4. Density profile of the plasma sheet.

uncertainty mainly due to shot-to-shot variation of laser power. In this case the peak plasma density $\left(n \approx 2 \times 10^{12}\right.$ $\mathrm{cm}^{-3}$ ) with our laser beam is barely above the critical density required for total reflection of $\mathrm{X}$-band microwaves.

To obtain a high reflection coefficient and maintain a relatively uniform axial plasma profile over multiple microwave wavelength distances, we perform most of the plasmamicrowave interaction measurements at the base pressure of 60-70 m'Torr.

\section{B. Plasma sheet thickness analysis}

Figure 4 illustrates the Langmuir probe measured plasma density profile in the dimension perpendicular to the Iaser beam $(y)$ direction. The plasma clearly demonstrates a "sheet" profile with a high density region of $4 \mathrm{~mm}$ thickness, and a very large density gradient outside this region. The 4 $\mathrm{mm}$ thickness is determined by the input laser beam cross section. It has been shown previously ${ }^{3}$ that the plasmamicrowave interaction is strongly influenced by the plasma profile gradient. In our experiment it is desirable to obtain a high density plasma core with a sharp edge gradient since it most closely resembles a conducting sheet for microwave reflection.

The measurement of plasma temperature in this region is illustrated in Fig. 5. The peak temperature is $T_{e}=0.8 \mathrm{eV}$. The temperature profile, as measured by a triple-tip probe, is much flatter. This can be attributed to the fact that the tripleprobe measurement is very sensitive to the high energy tail of the distribution function. A small amount of "hot" electrons are weighted more than the bulk "cold" electrons in the measured value of temperature. The experimental error in the triple-probe measurement is relatively high $(\approx 25 \%){ }^{7}$

The electron-ion recombination process dominates the plasma dynamics in the decaying mode, as will be discussed further in Sec. IV, thus very little expansion of the plasma sheet is expected. In Fig. 4 the plasma density remains within a $4 \mathrm{~mm}$ region with very little expansion observed. This result is significant in that as long as the density is above the critical density for total reflection, an the plasma 


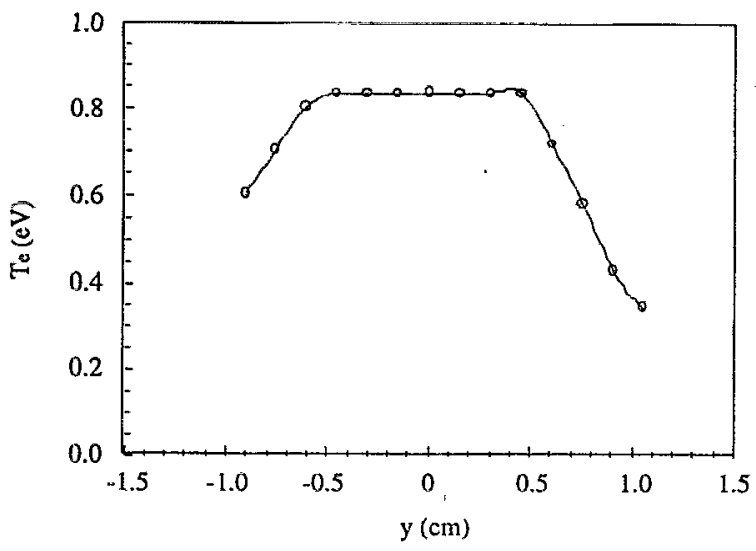

FIG. 5. Plasma temperature profile as measured by a triple probe.

sheet has sufficient thickness, the rapid plasma decay will have very little effect on the reflected microwave amplitude and phase.

\section{THEORETICAL AND NUMERICAL MODELING}

The plasma formation, plasma profile evolution, and decay after the laser beam is turned off can be described by a one-dimensional differential equation with the neglect of axial diffusion $(\partial / \partial z=0)$ for these time scales

$$
\frac{\partial n_{e}}{\partial t}=D_{a} \frac{\partial^{2} n_{e}}{\partial y^{2}}-\alpha_{r} n_{e}^{2}
$$

where $D_{a}$ is the ambipolar diffusion coefficient and $\alpha_{r}$ is the electron-ion recombination coefficient. We neglect the attachment term $\left(\alpha_{a} n_{e}\right)$ since this term is usually very small compared to the rest of the terms for the high density plasma conditions considered.

Assuming that diffusion is small, as is the case for weakly ionized plasmas, the solution of Eq. (2) becomes

$$
\frac{1}{n}=\frac{1}{\left.n\right|_{t=0}}+\alpha_{r} t
$$

Thus the recombination coefficient can be derived from the slope of a linear relation between $1 / n$ and time. From Fig. 6 it is shown that the relation is close to a linear one. This provides evidence that the recombination term in Eq. (2), $\alpha_{r} n_{e}^{2}$, dominates. The slope of this linear relation yields $\alpha_{r}=1.7 \times 10^{-7} \mathrm{~cm}^{3} \mathrm{~s}^{-1}$.

A study of the time variation of the half-width of the plasma sheet further supports the notion that the plasma is dominated by recombination for $t \leqslant 1.2 \mu$ s and that diffusion becomes significant after $1.2 \mu \mathrm{s}$. A simple dimensional analysis indicates that if the plasma is in a pure diffusive state, the half-width $\Delta \sim \sqrt{t}$, while a pure recombination process in the plasma yields $\Delta \sim \sqrt{\ln (t)}$. So, the increase of $\Delta$ due to recombination is much slower than that due to diffusion.

Figure 7 shows the time variation of the measured plasma sheet half-width. The increase in $\Delta$ with time is very small when $t \leqslant 1.2 \mu \mathrm{s}$. This is consistent with the result in Fig. 6 when the plasma is dominated by the recombination

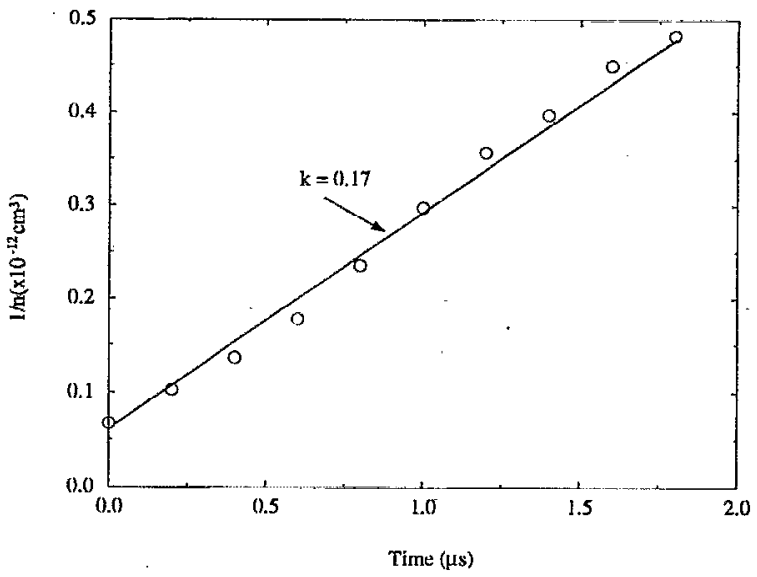

FIG. 6. $1 / n$ vs time at the plasma center.

process. When $t \geqslant 1.2 \mu \mathrm{s}$, on the other hand, the increase in $\Delta$ with time becomes faster. Since recombination is proportional to $n^{2}$ while the diffusion is proportional to $n$, one expects that the diffusion term becomes more significant as the peak density decreases.

The calculation of the diffusion coefficient $D_{a}$ from the measured data requires solving Eq. (2). The equation can be simplified for early times $(t \leqslant 1.2 \mu \mathrm{s})$ by treating the diffusion term as a perturbation superimposed upon the dominant recombination process. Assuming $D_{a} \sim \epsilon D$ and $n_{e}=n_{e 0}$ $+\epsilon n_{e 1}$, where $\epsilon$ is a small parameter, in Eq. (2), we have for zeroth order

$$
\frac{\partial n_{e 0}(y, t)}{\partial t}=-\alpha_{r} n_{e 0}^{2}(y, t)
$$

and for the first order

$$
\frac{\partial n_{e 1}(y, t)}{\partial t}=D \frac{\partial^{2} n_{e 0}(y, t)}{\partial y^{2}}-2 \alpha_{r} n_{e 0}(y, t) n_{e 1}(y, t) .
$$

The solution to Eq. (4) is

$$
n_{e 0}(y, t)=\frac{N(y)}{N(y) \alpha_{r} t+1} .
$$

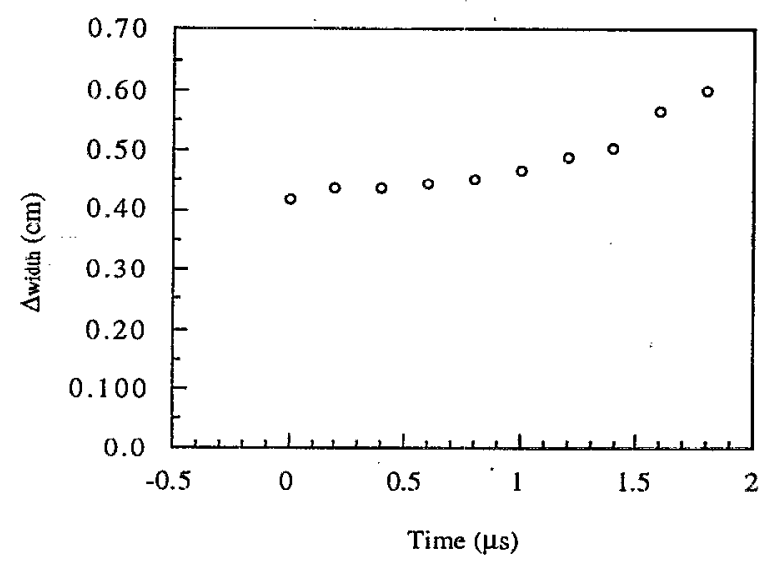

FIG. 7. Plasma sheet half-width vs time. 

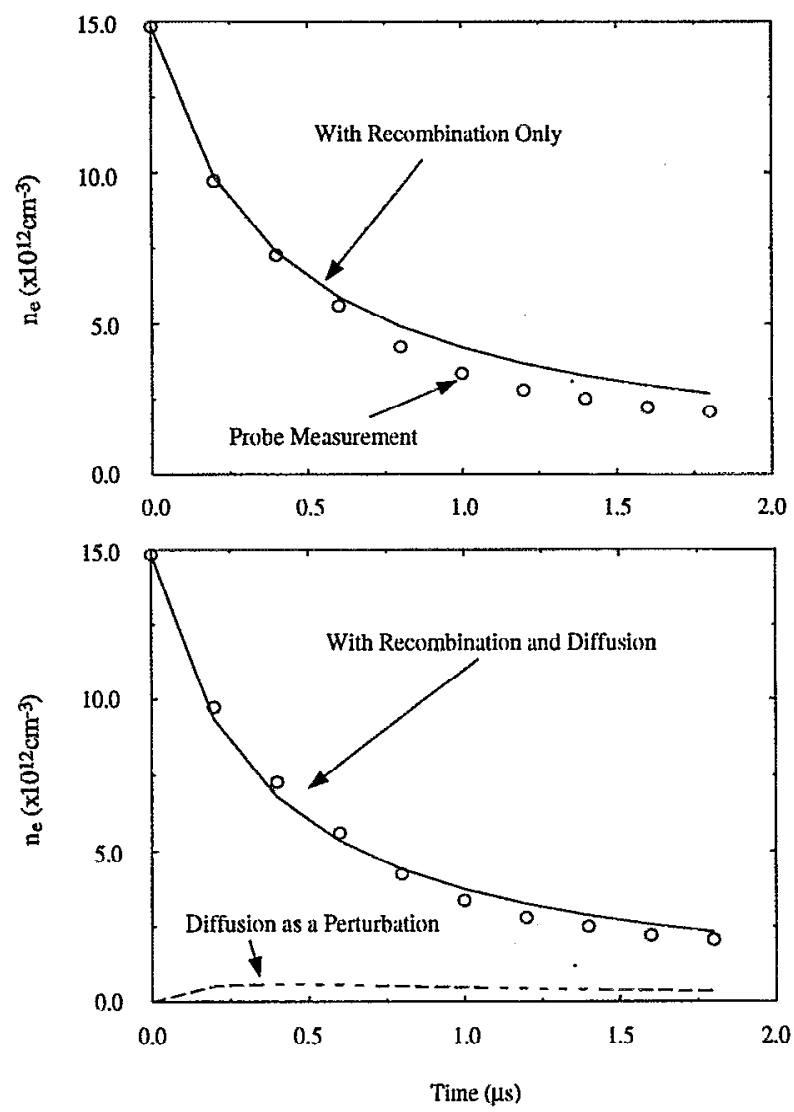

FIG. 8. Comparison of the simulation with the measured results.

Substituting the solution for $n_{e 0}(y, t)$ into Eq. (5) and expanding the second-order derivative term we obtain

$$
\frac{\partial^{2} n_{e 0}}{\partial y^{2}}=\frac{\partial N}{\partial y} \frac{\partial}{\partial y}\left[\frac{1}{\left(N \alpha_{r} t+1\right)^{2}}\right]+\frac{1}{\left(N \alpha_{r} t+1\right)^{2}} \frac{\partial^{2} N}{\partial y^{2}} .
$$

At $y=0$ we take $\partial N /\left.\partial y\right|_{y=0}=0, \partial^{2} N /\left.\partial y^{2}\right|_{y=0}=A$, and $\left.N\right|_{y=0}=N_{0}$, which gives

$$
\frac{\partial n_{e 1}(0, t)}{\partial t}=\frac{A D}{\left(N_{0} \alpha_{r} t+1\right)^{2}}-\frac{2 \alpha_{r} N_{0}}{\left(N_{0} \alpha_{r} t+1\right)} n_{e 1}(0, t) .
$$

The solution is (assuming $\left.n_{e 1}\right|_{t=0}=0$ ) as follows:

$$
n_{e 1}(0, t)=\frac{A D t}{\left(N_{0} \alpha_{r} t+1\right)^{2}} \text {. }
$$

Therefore, the electron density at $y=0$ is

$$
n_{e}(0, t)=\frac{N_{0}}{N_{0} \alpha_{r} t+1}+\frac{A D_{a} t}{\left(N_{0} \alpha_{r} t+1\right)^{2}} .
$$

Figure 8 illustrates the comparison of the plasma decay process obtained by measurement and described by Eq. (10). The best fit corresponds to $D_{a}=2.8 \pm 0.4 \times 10^{4} \mathrm{~cm}^{2} \mathrm{~s}^{-1}$ and $\alpha_{\mathrm{r}}=1.8 \pm 0.1 \times 10^{-7} \mathrm{~cm}^{3} \mathrm{~s}^{-1}$. The value of $A$ is calculated assuming a Gaussian density distribution at $t=0$ and is negative for a center-peaked profile.

To verify the perturbation expansion, we estimate the two terms on the right-hand side of Eq. (2). With $n(0,0.1$ $\mu \mathrm{s}) \approx 1.5 \times 10^{13} \mathrm{~cm}^{-3}$ and a Gaussian distribution, the recom- bination term is 10 times larger than the diffusion term at $t=0.1 \mu \mathrm{s}$. Since the recombination term is proportional to $n^{2}$ while the diffusion term is proportional to $n$ only, we expect the diffusion perturbation to become significant when $n \leqslant\left. 0.1 n\right|_{t=0}$.

Electron collisions with neutral particles are the dominant process, thus we can estimate the mean-free path for ion-neutral collisions using the classical expression: $\bar{\lambda}=k T$ I $\left(\sqrt{2} \pi d_{i}^{2} p\right)$ where $d_{i}$ is the diameter of the molecule and $p$ is the pressure. Using $T_{i}=0.025 \mathrm{eV}$ (room temperature), $p=60$ mTorr, and $d_{i}=(208)^{1 / 3} \times d_{\mathrm{H}_{2}}$, we have $\bar{\lambda}=3.37 \times 10^{-4} \mathrm{~m}$. The collision frequency becomes $\nu_{i n}=\bar{v}_{i} / \bar{\lambda}=4.52 \times 10^{5} \mathrm{~s}^{-1}$. This gives $D_{i}=2.65 \times 10^{2} \mathrm{~cm}^{2} \mathrm{~s}^{-1}$. The value of $D_{a}$ can be estimated from the ambipolar condition: $D_{a}=D_{i}\left(1+T_{e} / T_{i}\right)$ which yields $D_{a} \approx 1.1 \times 10^{4} \mathrm{~cm}^{2} \mathrm{~s}^{-1}$. This estimate is $60 \%$ smaller than the $D_{a}$ obtained as a best fit to Eq. (10). The accuracy of the calculation is limited by the simplified model and uncertainties in the parameter $d_{i}$ for TMAE.

\section{SUMMARY}

Studies of a laser-produced sheet plasma for use as a microwave agile mirror are carried out. We have measured the cross section of the sheet plasma density and temperature. A "sheet-like" plasma profile is obtained and sustained for a period much longer $\left(\tau_{p} \geqslant 2 \mu \mathrm{s}\right)$ than the laser pulse $\left(\tau_{L}=17 \mathrm{~ns}\right)$. The axial attenuation of the plasma density due to photon absorption is measured with various filling pressures of the working gas. The plasma decay after the formation is found to be dominated by the charge recombination process. A plasma dynamic model which includes a diffusion and recombination process is studied. We use a perturbation method to solve the model equation and to compare the diffusion coefficient with the theoretical estimate based on a collisional model. The measured diffusion coefficient is 2.5 times that obtained by our estimate.

We are able to obtain high density $\left(n_{e}=2 \times 10^{13} \mathrm{~cm}^{-3}\right)$, low temperature $\left(T_{e}=0.8 \mathrm{eV}\right)$ plasmas over moderate sheet dimensions ( $30 \mathrm{~cm} \times 8 \mathrm{~cm} \times 4 \mathrm{~mm}$ ) by this VUV laser formation technique. This plasma sheet will be used to study microwave reflection, transmission, and scattering properties for use as an agile mirror and the measurements will be presented in a future paper.

\section{ACKNOWLEDGMENTS}

The authors wish to thank Dr. M. Bettenhausen and Y. Mouzouris for valuable discussions on the numerical modeling. This work is supported by AFOSR Grant No. F4962094-1-0054 and AFOSR AASERT Award No. F49620-93-10465 .

${ }^{1}$ R. J. Vidmar, IEEE Trans. Plasma Sci. 18, 733 (1990)

${ }^{2}$ W. Manheimer, IEEE Trans. Plasma Sci. 19, 1228 (1991).

${ }^{3}$ K. R. Stalder, R. J. Vidmar, and D. J. Eckstrom, J. Appl. Phys. 72, 5089 (1992).

${ }^{4}$ R. A. Meger, J. Mathew, J. A. Gregor, R. E. Pechacek, R. F. Fernsler, 
W. Manheimer, and A. E. Robson, Phys. Plasma 2, 2532 (1995).

${ }^{5}$ Y. Zhang and J. E. Scharer, J. Appl. Phys. 73, 4779 (1993).

'J. E. Scharer, O. C. Eldridge, S. F. Chang, Y. S. Zhang, M. Bettenhausen, and N. T. Lam, IEEE Trans. Plasma Sci. 21, 271 (1993).
${ }^{7}$ S.-L. Chen and T. Sekiguchi, J. Appl. Phys. 36, 2363 (1965).

${ }^{8}$ D. Anderson, IEEE Trans. Nucl. Sci. NS-28, 842 (1981).

${ }^{9}$ R. A. Holroyd, J. M. Preses, C. L. Woody, and R. A. Johnson, Nucl. Instrum. Methods Phys. Res. A 261, 440 (1987). 